\title{
GENERALISED IMPEDANCE CONVERTERS WITH ONLY TRANSCONDUCTANCE ELEMENTS AND GROUNDED CAPACITORS
}

\author{
IQBAL A. KHAN ${ }^{\mathrm{a}, *}$, MUSLIM T. AHMED ${ }^{\mathrm{b}}$ and NIGAR MINHAJ ${ }^{\mathrm{c}}$ \\ ${ }^{a}$ Department of Electronics Engineering, Aligarh Muslim University, Aligarh 202 002, India; \\ ${ }^{\mathrm{b}}$ Department of Electrical and Electronics Engineering, University of Bahrain, Isa Town, Bahrain; \\ ${ }^{\mathrm{c}}$ Electronics Engineering Section, Women's Polytechnic, Aligarh Muslim University, \\ Aligarh 202 002, India
}

(Received 18 May 2002; In final form 3 July 2002)

\begin{abstract}
Novel generalised impedance converters are presented, which use only transconductance elements and/or grounded capacitors for the realisation of a wide variety of immittance functions. The generalised impedance converters can be used in the realisation of low sensitivity continuous time filters. The use of transconductance elements and grounded capacitors in the realisation of continuous time filters lend to electronic tunability and compatibility to integration in contemporary IC technologies. The generalised impedance converters are also verified using PSPICE-based simulation.
\end{abstract}

Keywords: Generalized impedance converters; Transconductance element; $\mathrm{g}_{m}-\mathrm{C}$ immittance simulation

\section{INTRODUCTION}

In recent years, there has been a great interest in the design of integrable continuous time filters using transconductance elements and grounded capacitors (TGC). The TGC circuits possess superior high frequency performance and economize the chip area requirements as compared with OTA-C circuits. The TGC circuit provides the attractive implementation of continuous time analog filters in the CMOS technology [1-7].

In this paper, novel TGC based generalised impedance converters (GICs) are presented. The GICs use only transconductance elements (TEs) and grounded capacitors in their realisations. The GICs are capable of realising a wide variety of ideal immittance simulators. These ideal simulators can be employed for the realisation of integrable low-sensitivity continuous time filters in CMOS technology.

\section{CIRCUIT REALISATION}

The basic scheme for realising the generalised impedance converters is shown in Figure 1. The scheme is basically used for converting a two port network having voltage transfer

* Corresponding author. 


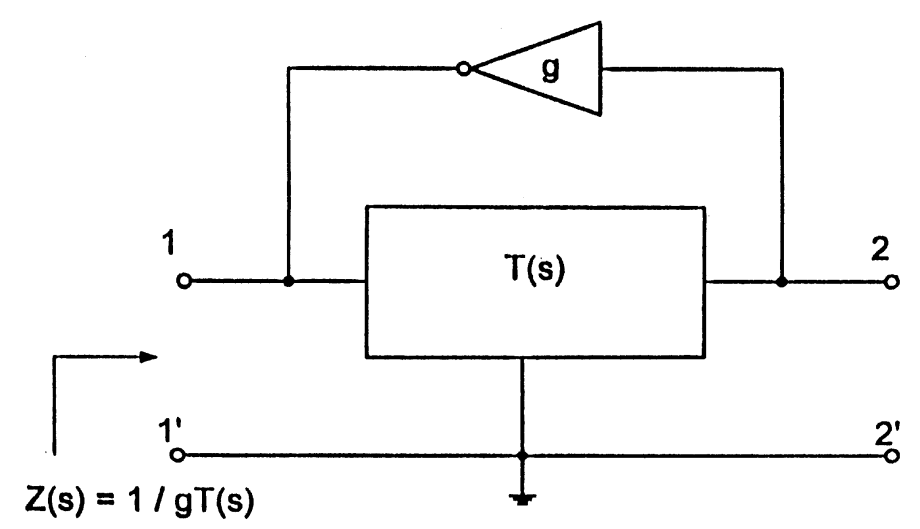

FIGURE 1 Basic scheme for the GIC realisation.

function $T(s)$ and infinite input impedance into grounded immittance simulator, where impedance function at port $1-1^{\prime}$ is given by $[1,8]$

$$
Z_{\text {in }}(s)=\frac{V}{I}=\frac{1}{g T(s)}
$$

where, $g$ is the transconductance gain of the feedback transconductor. The scheme of Figure 1, has been used to realise a novel generalised impedance converter as shown in Figure 2. The voltage transfer function block $T(s)$ is realised by cascading four voltage gain blocks having voltage gain $K_{1}, K_{2}, K_{3}$ and $K_{4}$ respectively, and expressed as

$$
T(s)=K_{1} K_{2} K_{3} K_{4}
$$

where $K_{1}=-g_{1} Z_{1}, K_{2}=1 / g_{2} Z_{2}, K_{3}=-g_{3} Z_{3}$ and $K_{4}=1 / g_{4} Z_{4}$. Thus the resulting input impedance function of the GIC of Figure 2 can be given as

$$
Z_{\text {in }}(s)=\frac{g_{2} g_{4}}{g g_{1} g_{3}} \frac{Z_{2} Z_{4}}{Z_{1} Z_{3}}
$$

By appropriate selection of $Z_{1}, Z_{2}, Z_{3}$ and $Z_{4}$, the GIC can realise ideal grounded inductor, resistor/capacitor multiplier, frequency dependent negative resistor (FDNR) and super inductor [8]. It is to be noted that all the passive elements are in grounded form. Obviously if any

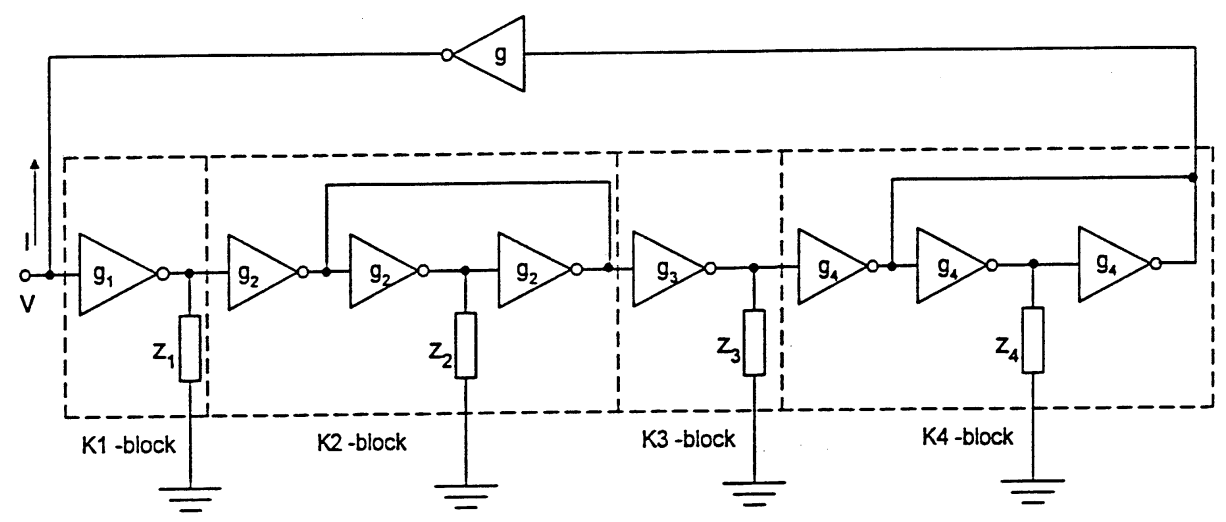

FIGURE 2 The complete GIC. 
TABLE I Realisation of TGC-based GICs.

\begin{tabular}{lccl}
\hline S.No. & $T(s)$ & $Z_{\text {in }}(s)$ & \multicolumn{1}{c}{ Type of functions available } \\
\hline 1 & 1 & $\frac{1}{g}$ & Resistor \\
2 & $K_{2}$ & $\frac{g 2}{g} Z_{2}$ & Resistor, capacitor \\
3 & $K_{1} K_{2}$ & $-\frac{g_{2}}{g g_{1}}\left(Z_{2} / Z_{1}\right)$ & Negative resistor, negative capacitor \\
4 & $K_{3} K_{4}$ & $-\left(\frac{g_{4}}{g g_{3}}\right)\left(Z_{4} / Z_{3}\right)$ & Negative resistor, negative capacitor \\
5 & $K_{1} K_{3}$ & $\frac{1}{g g_{1} g_{3}}\left(1 / Z_{1} Z_{3}\right)$ & Super inductor \\
6 & $K_{2} K_{4}$ & $\frac{g_{2} g_{4}}{g}\left(Z_{2} Z_{4}\right)$ & FDNR \\
7 & $K_{1} K_{2} K_{3}$ & $\frac{g_{2}}{g g_{1} g_{3}}\left(Z_{2} / Z_{1} Z_{3}\right)$ & Inductor/super inductor \\
8 & $K_{1} K_{3} K_{4}$ & $\frac{g_{4}}{g g_{1} g_{3}}\left(Z_{4} / Z_{1} Z_{3}\right)$ & Inductor/super inductor \\
9 & $K_{1} K_{2} K_{4}$ & $-\frac{g_{2} g_{4}}{g g_{1}}\left(Z_{2} Z_{4} / Z_{1}\right)$ & Negative resistor, negative capacitor, \\
10 & $K_{2} K_{3} K_{4}$ & $-\frac{g_{2} g_{4}}{g g_{3}}\left(Z_{2} Z_{4} / Z_{3}\right)$ & negative inductor, FDPR \\
11 & $K_{1} K_{2} K_{3} K_{4}$ & $\frac{g_{2} g_{4}}{g g_{1} g_{3}}\left(Z_{2} Z_{4} / Z_{1} Z_{3}\right)$ & Negative resistor, negative capacitor, \\
negative inductor, FDPR
\end{tabular}

of these happen to be a resistor, it can conveniently be replaced by a TE-based grounded resistor simulator [2]. Thus the resulting GIC will be in TGC form.

It is to be noted that all the four K-blocks may not be required to simulate a single impedance function. The useful combinations of K-blocks to implement the transfer function block-T for the realisation of various GICs are given in Table I. It is clear from the table that the basic scheme can be used to realise a variety ideal component simulators, including negative resistor, negative capacitor, negative inductor, frequency dependent negative resistor (FDNR), frequency dependent positive resistor (FDPR) as well as the super inductor, through appropriate selection of passive $\mathrm{R}, \mathrm{C}$ elements.

\section{SIMULATION RESULTS}

To verify the theory the prototype band-pass filter (BPF) of Figure 3(a) is transformed into its FDNR version, as shown in Figure 3(b). The ideal FDNR in Figure 3(b) is replaced by the

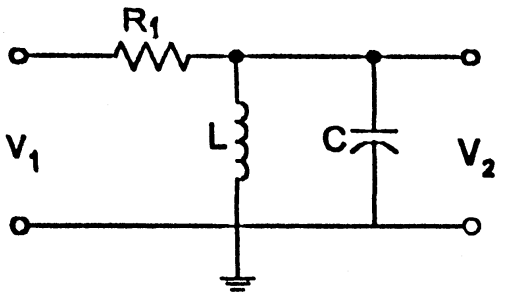

(a)

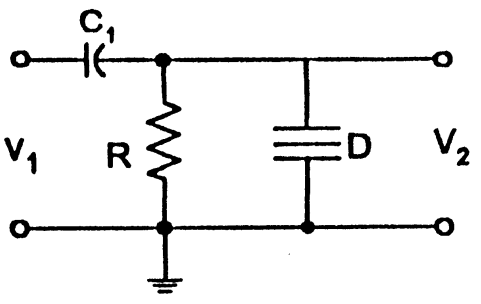

(b)

FIGURE 3 (a) Prototype second-order Band Pass Filter (BPF); (b) Transformed FDNR-based version of BPF of (a). 


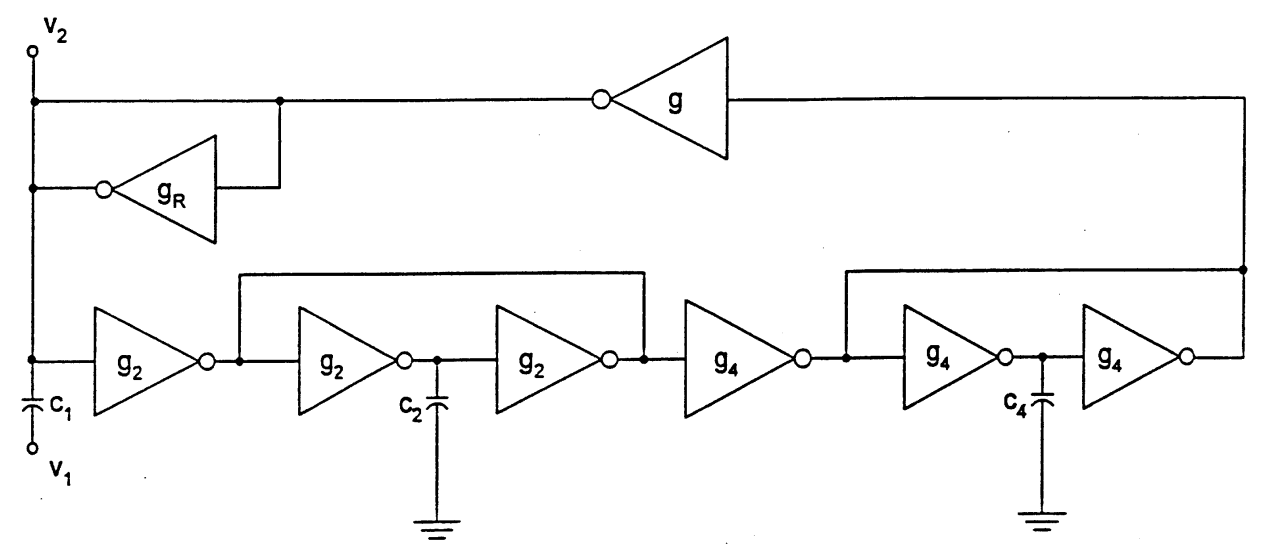

FIGURE 4 TE-based version of BPF of Figure 3(b).

simulated FDNR obtained from the GIC of Table I (S.No. 6), and the grounded resistor by the TE-based resistor of Table I [2]. The resulting BPF is shown in Figure 4, with $R=1 / g_{R}$ and $D=\left(g C_{2} C_{4}\right) /\left(g_{2} g_{4}\right)$. The filter's pole- $\omega_{0}$ and pole- $Q$ respectively can be expressed as

$$
\omega_{0}=\left(\frac{g_{R} g_{2} g_{4}}{g C_{2} C_{4}}\right)^{1 / 2}, \quad Q=\frac{1}{C_{1}}\left(\frac{g_{R} g C_{2} C_{4}}{g_{2} g_{4}}\right)^{1 / 2}
$$

Assuming all TEs identical (i.e. $g_{2}=g_{4}=g_{R}=g$ ), and $C_{2}=C_{4}=C$, the pole- $\omega_{0}$ and pole- $Q$ respectively of this $\mathrm{BPF}$ reduces to:

$$
\omega_{0}=\frac{g}{C} \quad \text { and } \quad Q=\frac{C}{C_{1}}
$$

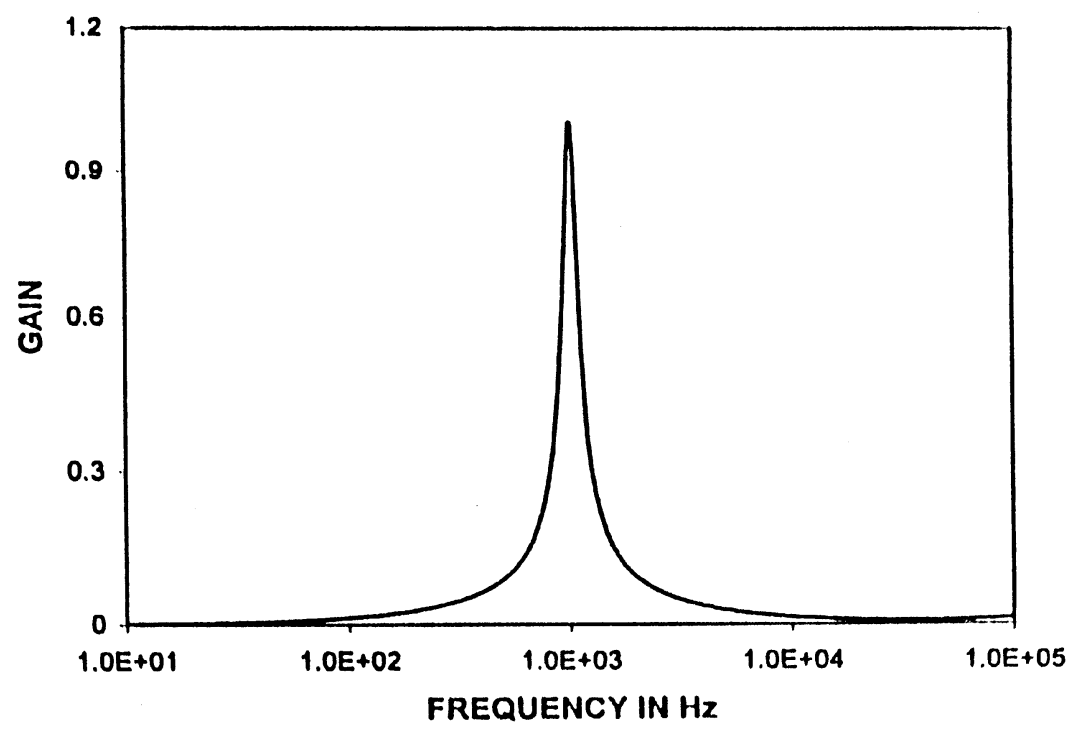

FIGURE 5 Frequency response curve of designed BPF of Figure 4. 
The BPF was designed for a centre frequency $f_{0}=1 \mathrm{kHz}$, and $Q=5$. Assuming $g=46 \mu \mathrm{mho}$, Eq. (5) results the component values as $C_{1}=1.464 \mathrm{nF}$, and $C=7.321 \mathrm{nF}$. The PSPICE model of PMOS and NMOS of Ref. [2], was considered to implement the TEs with gate voltages of $3.2 \mathrm{~V}$ in magnitude to set the $g_{m}=46 \mu$ mho. The simulation results of the BPF using PSPICE is shown in Figure 5, which demonstrate a close agreement with the theory.

\section{CONCLUSION}

A simple scheme for the realisation of generalised impedance converters using transconductance elements and grounded capacitors is presented. The scheme can conveniently provide the realisation of various types of ideal impedance function simulators including negative components, super inductor and FDPR. The use of grounded capacitors and transconductance elements is attractive for monolithic implementation of the GICs in MOS-technology. One of the GICs is verified using PSPICE-based simulation with good results.

\section{References}

[1] Tan, M. A. and Schaumann, R. (1988). Design of general biquadratic filters with only transconductances and grounded capacitors. I.E.E.E. Trans., CAS-35, 478-480.

[2] Tan, M. A. and Schaumann, R. (1989). Simulating general parameter LC-ladder filters for monolithic realisation with only transconductance elements and grounded capacitors. I.E.E.E. Transactions on Circuits and Systems, 36, 299-307.

[3] Schaumann, R. (1989). Design of continuous-time fully integrated filters. A review. IEE Proceedings of the Institution of Electrical Engineers. Pt. G, 136, 184-190.

[4] Sun, Y. and Filder, J. K. (1995). Resonator based universal OTA-grounded capacitor filter. International Journal of Circuit Theory and Applications, 23, 261-265.

[5] Sun, Y. and Filder, J. K. (1997). Structure generation and design of multiple loop feedback OTA-grounded capacitor filters. I.E.E.E. Transactions on Circuits and Systems, 44, 1-11.

[6] Efthivoulidis, G., Toth, L. and Tsividis, Y. P. (1998). Noise in $\mathrm{g}_{\mathrm{m}}-\mathrm{C}$ filters. I.E.E.E. Transactions on Circuits and Systems-II Analog and Digital Signal Processing, 45(3), 295-302.

[7] Efthivoulidis, G., Toth, L. and Tsividis, Y. P. (1998). OTA-C filter design using inductor substitution and bruton transformation methods. Electronics Letters, 34(22), 2082-2083.

[8] Khan, I. A., Ahmed, M. T. and Minhaj, N. (1992). Novel technique for immitance simulation-realisation of some all-active simulators. Int. J. Electronics, 72(3), 431-441. 

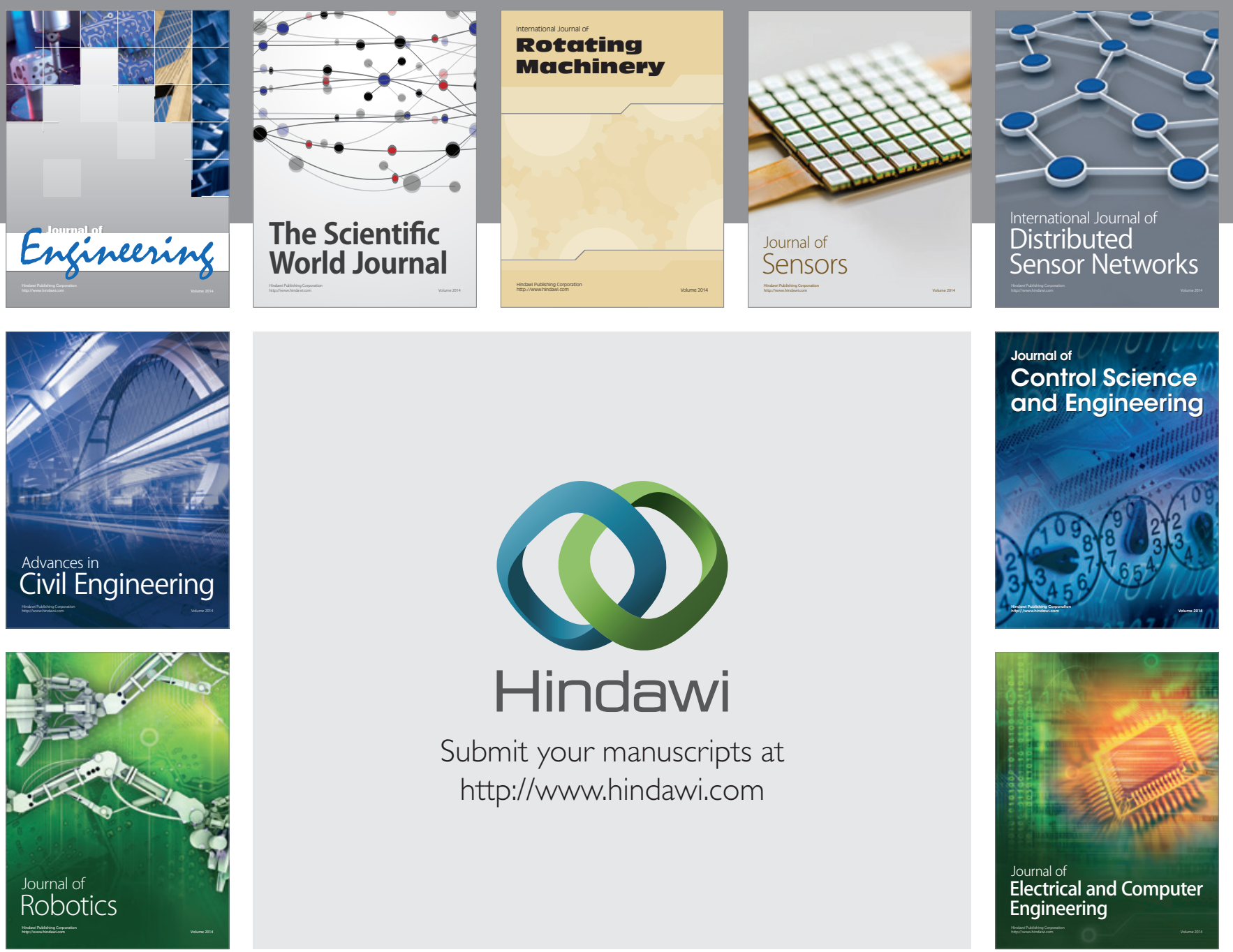

Submit your manuscripts at

http://www.hindawi.com
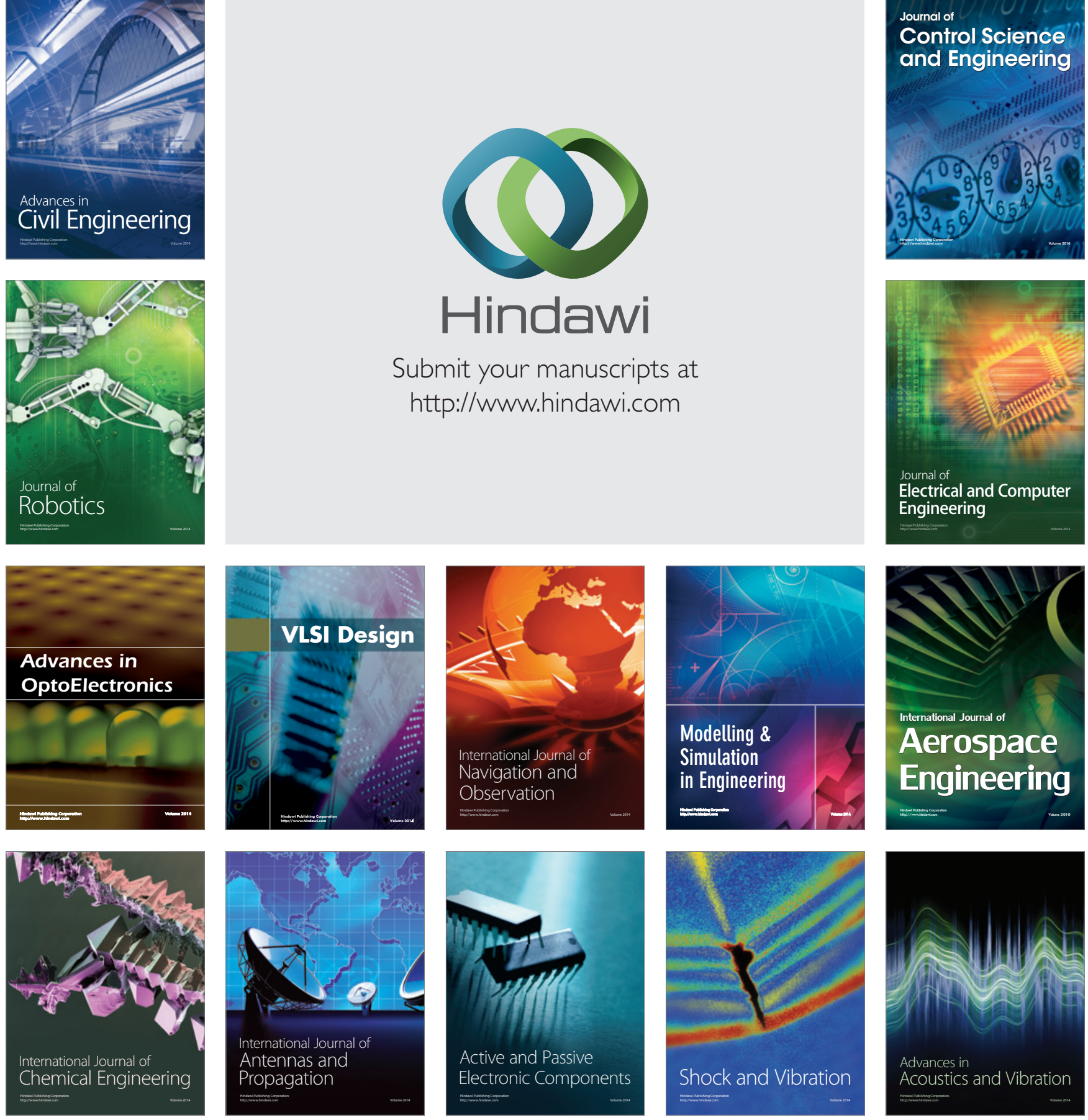